\title{
Minimization of the ground state of the mixture of two conducting materials in a small contrast regime
}

\author{
C. Conca, M. Dambrine, R. Mahadevan and D. Quintero
}

\begin{abstract}
We consider the problem of distributing two conducting materials with a prescribed volume ratio in a given domain so as to minimize the first eigenvalue of an elliptic operator with Dirichlet conditions. The gap between the two conductivities is assumed to be small (low contrast regime). For any geometrical configuration of the mixture, we provide a complete asymptotic expansion of the first eigenvalue. We then consider a relaxation approach to minimize the second order approximation with respect to the mixture. We present numerical simulations in dimensions two and three.
\end{abstract}

\section{Introduction}

Problems of mininimizing the ground state of composite materials appear frequently and are of interest in applications. We refer to Henrot [13, Cox and McLaughlin [7, 8], Cox and Lipton [6] and included references. In this article, we consider the following problem. Given a domain $\Omega$ and a subdomain $B$ and two nonnegative numbers $\alpha$ and $\beta$, we define the ground state $\lambda(B)$ of the mixture as the infimum of the $\lambda$ such that there exists $0 \neq u$ such that

$$
-\operatorname{div}\left(\left(\alpha+(\beta-\alpha) \chi_{B}\right) \nabla u\right)=\lambda u \text { in } \Omega \text { and } u=0 \text { on } \partial \Omega .
$$

In other words, $\lambda(B)$ is the smallest eigenvalue of the operator $-\operatorname{div}\left(\left(\alpha+(\beta-\alpha) \chi_{B}\right) \nabla\right.$. $)$ on $\mathrm{H}_{0}^{1}(\Omega)$. We are then interested in minimizing $\lambda(B)$ with respect to $B$ among the subdomains of $\Omega$ of given volume.

In general, it is well-known that this problem is not wellposed: the infimum is not usually reached at a given $B$ and we have to consider a relaxed version corresponding to a situation of homogenization (see [6]).

Nevertheless, when $\Omega$ is a ball, the infimum is reached on a radially symmetric domain $B^{*}$ (see [2, [4]). In the recent years, much attention has been put on the determination of the corresponding $B^{*}$. First, Conca and al. conjectured in [5] that the global minimizer $B^{*}$ in $\Omega$ should be a concentric ball of the prescribed volume. The conjecture was motivated by the situation in dimension one and by numerical simulations. Then, Dambrine and Kateb reinforced the conjecture by an order two sensitivity analysis in [9] by proving that the concentric ball of prescribed volume is a local strict minimizer of $\lambda(B)$.

However, Conca et. al. proved in 3 that the conjecture is false. Their strategy was the following. They consider the case of small contrast, that is to say, $\alpha$ and $\beta$ such that the difference of both conductivities is small: $\beta=\alpha(1+\varepsilon)$ and provide the first order asymptotic expansion $\lambda_{1}(B)$ of $\lambda(B)$ with respect to the small parameter $\varepsilon$ for any admissible domain $B \subset \Omega$. Then, they minimize the new objective functional $\lambda_{1}(B)$ with respect to $B$ and observe that the minimizer $B_{1}$ of this approximation is not always the concentric ball of prescribed volume. Finally, thanks to a precise estimate of the remainder in the approximation, they prove that $\lambda\left(B_{1}\right)<\lambda\left(B^{*}\right)$.

Finally, Laurain proved in [14] that the global minimum of the first eigenvalue in low contrast regime is either a centered ball or the union of a centered ball and of a centered ring touching the boundary, 
depending on the prescribed volume ratio between the two materials. Thus the small contrast case is well understood when the domain is a ball.

We aim in this work to make a precise analysis of the small contrast case in general domains. In Section 2 , to begin with, we characterize completely the full asymptotic expansion of $\lambda(B)$ with respect to the small parameter $\varepsilon$. Subsequently, we obtain a second order approximation $\lambda_{2}(B)$ of $\lambda(B)$ with uniform estimates for the remainder, uniform with respect to $B$. This means that minimizers for the second order approximation $\lambda_{2}(B)$ are approximate minimizers for the original objective functional $\lambda(B)$. With this motivation, in Section 3, we study the problem of minimizing $\lambda_{2}$. Unlike the first order approximation $\lambda_{1}(B)$, the minimization problem for $\lambda_{2}(B)$ is not, a priori, well posed and thus, qualitatively, resembles more closely the minimization problem for $\lambda(B)$. A relaxed formulation for the minimization problem for $\lambda_{2}(B)$ is obtained using $H$-measures. It can be seem that the relaxed problem for $\lambda_{2}(B)$ has a much more simple aspect compared to the relaxed problem for $\lambda(B)$ obtained in Cox and Lipton [6]. Finally, in Section 4, the optimality conditions for the relaxed problem for $\lambda_{2}(B)$ are obtained and the minimization problem is studied numerically using a descent algorithm.

\section{Asymptotic expansion of the first eigenvalue with respect to the constrast.}

We consider the low contrast regime, that is to say, $\alpha$ and $\beta$ such that the difference of both conductivities is small: $\beta=\alpha(1+\varepsilon)$. We shall denote the first eigenvalue in the problem (1.1) by $\lambda_{\varepsilon}(B)$ for a given distribution $B$ of the material with conductivity $\beta$ and a given value of the contrast parameter $\varepsilon>0$.

The existence of an asymptotic development for $\lambda_{\varepsilon}(B)$, for given $B$, is classical from perturbation theory of simple eigenvalues. By the Krein-Rutman theorem, the first eigenvalue $\lambda_{\varepsilon}(B)$ in (1.1) is simple. The corresponding normalized eigenfunction, with unit $L^{2}$ norm and taken to be non-negative, will be denoted by $u_{\varepsilon}(B)$. So, by classical results from perturbation theory (see, for instance, Theorem 3, Chapter 2.5 of Rellich [15] ), for a given $B$, the map $\varepsilon \mapsto\left(\lambda_{\varepsilon}, u_{\varepsilon}\right)$ is analytic in $\left(\mathbb{R}, \mathrm{H}_{0}^{1}(\Omega)\right)$. Therefore there are sequences $\left(\lambda_{i}\right)$ of real numbers and $\left(u_{i}\right)$ of functions in $\mathrm{H}_{0}^{1}(\Omega)$ such that:

$$
\lambda_{\varepsilon}=\sum_{i=0}^{\infty} \lambda_{i} \varepsilon^{i} \text { and } u_{\varepsilon}=\sum_{i=0}^{\infty} u_{i} \varepsilon^{i}
$$

As a consequence, there are constants $C_{n}(B)$ such that

$$
\left|\lambda_{\varepsilon}-\sum_{i=0}^{n} \lambda_{i} \varepsilon^{i}\right| \leq C_{n}(B) \varepsilon^{n+1} \text { and }\left\|u_{\varepsilon}-\sum_{i=0}^{n} u_{i} \varepsilon^{i}\right\|_{\mathrm{H}_{0}^{1}} \leq C_{n}(B) \varepsilon^{n+1} .
$$

In this section, we will first identify the coefficients $\lambda_{i}, u_{i}$ then prove that the constants $C_{n}(B)$ can be taken uniform in $B$. This will serve in obtaining an approximate model problem for the eigenvalue minimization problem. 


\subsection{Computation of the coefficients in 2.1}

The terms in the the asymptotic expansions in 2.1) may be identified, formally, by injecting the expansions in the equations defining $\left(\lambda_{\varepsilon}, u_{\varepsilon}\right)$, that is,

$$
\begin{aligned}
-\operatorname{div}\left(\alpha\left(1+\chi_{B} \varepsilon\right) \nabla\left(\sum_{i=0}^{\infty} u_{i} \varepsilon^{i}\right)\right) & =\left(\sum_{i=0}^{\infty} \lambda_{i} \varepsilon^{i}\right)\left(\sum_{i=0}^{\infty} u_{i} \varepsilon^{i}\right) \text { in } \Omega, \\
\sum_{i=0}^{\infty} u_{i} \varepsilon^{i} & =0 \text { on } \partial \Omega \\
\int_{\Omega}\left(\sum_{i=0}^{\infty} u_{i} \varepsilon^{i}\right)^{2} & =1 .
\end{aligned}
$$

and we obtain then the following relationships by identifying the coefficients of same order in the previous power series.

$$
\begin{gathered}
\left\{\begin{aligned}
-\alpha \Delta u_{0}-\lambda_{0} u_{0} & =0 \text { in } \Omega \\
u_{0} & =0 \text { on } \partial \Omega \quad \forall i \geq 0, \\
\int_{\Omega} u_{0}^{2} & =1
\end{aligned}\right. \\
\left\{\begin{aligned}
-\alpha \Delta u_{i}-\lambda_{0} u_{i}= & \operatorname{div}\left(\alpha \chi_{B} \nabla u_{i-1}\right)+\sum_{k=1}^{i} \lambda_{k} u_{i-k} \text { in } \Omega \quad \forall i \geq 1, \\
u_{i}= & 0 \text { on } \partial \Omega \quad \forall i \geq 0, \\
\sum_{k=0}^{i} \int_{\Omega} u_{k} u_{i-k}= & 0 \quad \forall i \geq 1
\end{aligned}\right.
\end{gathered}
$$

It is possible to rigorously justify the relations by using the expansions 2.1) in the weak formulation of the partial differential equation in (1.1). We then have an iterative procedure to compute the pair $\left(\lambda_{i}, u_{i}\right)$.

The case: $i=0$. By definition, one has:

$$
\begin{aligned}
-\alpha \Delta u_{0}-\lambda_{0} u_{0} & =0 \text { in } \Omega \\
u_{0} & =0 \text { on } \partial \Omega .
\end{aligned}
$$

Hence, the couple $\left(\lambda_{0}, u_{0}\right)$ is an eigenpair of $-\alpha \Delta$ with homogeneous Dirichlet boundary condition. Clearly $u_{0} \geq 0$ in $\Omega$ since $u_{\varepsilon} \rightarrow u_{0}$ as $\varepsilon \rightarrow 0$ and the eigenmodes $u_{\varepsilon}$ are non-negative. Now, by the Krein-Rutman theorem, since all eigenmodes change sign except those associated to the first eigenvalue, we obtain that $\lambda_{0}$ is the ground state of $-\alpha \Delta$ with Dirichlet boundary condition and $u_{0}$ is the positive eigenmode with $\mathrm{L}^{2}$-norm 1.

Now assume that, for a given $i$, we have knowledge of all the $\lambda_{k}, u_{k}$ for $k<i$. We now then treat The case $k=i$. We know that $u_{i}$ satisfies the equation

$$
\begin{aligned}
-\alpha \Delta u_{i}-\lambda_{0} u_{i} & =\operatorname{div}\left(\alpha \chi_{B} \nabla u_{i-1}\right)+\sum_{k=1}^{i} \lambda_{k} u_{i-k} \text { in } \Omega, \\
u_{i} & =0 \text { on } \partial \Omega
\end{aligned}
$$

Notice that the right hand side has the unknown quantity $\lambda_{i}$. We shall first obtain an expression for $\lambda_{i}$ in terms of $\lambda_{k}$ 's and $u_{k}$ 's for $k<i$ which have been assumed to be calculated previously. The compatibility 
condition, the Fredholm alternative for the equation (2.6), imposes the orthogonality of the right hand side of the former equation to the kernel of $-\alpha \Delta-\lambda_{0} I$ with Dirichlet boundary condition which is spanned by $u_{0}$

$$
\int_{\Omega}\left(\operatorname{div}\left(\alpha \chi_{B} \nabla u_{i-1}\right)+\sum_{k=1}^{i} \lambda_{k} u_{i-k}\right) u_{0}=0 .
$$

This gives the expression for the eigenvalue $\lambda_{i}$

$$
\lambda_{i}=\int_{B} \alpha \nabla u_{i-1} \cdot \nabla u_{0}-\sum_{k=2}^{i-1} \int_{\Omega} \lambda_{i-k} u_{0} u_{k}
$$

taking into account the fact that the $L^{2}$ norm of $u_{0}$ is 1 and, $u_{0}$ and $u_{1}$ are orthogonal. In the sequel, whenever there is a sum whose upper limit is less than the lower limit, we shall adopt the convention that the sum is 0 .

Now, to end, we note that $u_{i}$ is not completely determined by the equation (2.6), but only upto the kernel of $-\alpha \Delta-\lambda_{0} I$. For $i=0$, the non-negativity of $u_{0}$ and the normalization condition (the third relation in (2.2) ) determines uniquely $u_{0}$. For general $i$, having determined uniquely the $u_{k}$ for $k<i$, the term $u_{i}$ is determined uniquely using the normalization condition (the third relation in (2.3) which can be written as

$$
\int_{\Omega} u_{i} u_{0}=-\frac{1}{2} \sum_{k=1}^{i-1} \int_{\Omega} u_{k} u_{i-k}
$$

and should be understood as the orthogonality relation $\int_{\Omega} u_{i} u_{0}=0$ when $i=1$.

\subsection{Uniform estimate of the remainders}

We seek to estimate the remainder in the expansions (2.1), uniformly in $B$. Our main results in this section are the following estimates.

Proposition 2.1 There exists a constant $C$, independent of $B$, such that

$$
\left|\lambda_{\varepsilon}-\left(\lambda_{0}+\varepsilon \lambda_{1}\right)\right| \leq \sqrt{\frac{\lambda_{0}}{\alpha}} C \varepsilon^{2} .
$$

Proposition 2.2 There is a constant $C>0$ independent of $B$ such that:

$$
\left|\lambda_{\varepsilon}-\left(\lambda_{0}+\varepsilon \lambda_{1}+\varepsilon^{2} \lambda_{2}\right)\right| \leq 2 C \varepsilon^{3} \sqrt{\frac{\lambda_{0}}{\alpha}} .
$$

The main tool we use for the estimation of the remainders is the notion of $h$-quasimode with $h=\mathcal{O}\left(\varepsilon^{k}\right)$, for $k=1,2$ in the sequel. The notion of quasimode is defined as follows.

Definition 2.3 Let $A$ be a self-adjoint operator on a Hilbert space $H$ with domain $D(A)$. For a fixed $h>0$, a pair $(\lambda, u) \in \mathbb{R} \times D(A) \backslash\{0\}$ is called a h-quasimode if we have

$$
\|(A-\lambda) u\|_{H} \leq h\|u\|_{H} .
$$

The interest of such a definition relies on the following fact: if $(\lambda, u)$ is a $h$-quasimode of $A$, then the distance from $\lambda$ to the spectrum of $A$ is less than $h$ and the distance between $u$ and certain eigenspaces of $A$ can be estimated (See Lemma 2-2 in [10]). We will prove that our truncated power series expansions are quasimodes in the Hilbert space $\mathrm{H}^{-1}(\Omega)$. 


\section{Remainder of order one.}

The first step is to prove a uniform bound in $B$ of $\|u\|_{\mathrm{H}^{1}(\Omega)}$.

Lemma 2.4 There exists $C$, which is independent of $B$, such that:

$$
\left\|u_{1}\right\|_{\mathrm{H}_{0}^{1}(\Omega)} \leq C \text { and }\left|\lambda_{\varepsilon}-\lambda_{0}\right| \leq C \varepsilon
$$

Proof of Lemma 2.4 By using 2.7), with $i=1$, we have the following expression and uniform bounds for $\lambda_{1}(B)$

$$
\lambda_{1}=\int_{B} \alpha\left|\nabla u_{0}\right|^{2} \leq \alpha \int_{\Omega}\left|\nabla u_{0}\right|^{2}=\lambda_{0} .
$$

By (2.3), for $i=1, u_{1}$ satisfies the following:

$$
\begin{aligned}
-\alpha \Delta u_{1}-\lambda_{0} u_{1} & =-\operatorname{div}\left(\alpha \chi_{B} \nabla u_{0}\right) \text { in } \Omega, \\
u_{1} & =0 \text { on } \partial \Omega, \\
\int_{\Omega} u_{0} u_{1} & =0 .
\end{aligned}
$$

After multiplying the first relation by $u_{1}$ and integrating over $\Omega$, by integration by parts, we get

$$
\int_{\Omega} \alpha\left|\nabla u_{1}\right|^{2}-\lambda_{0} \int_{\Omega} u_{1}^{2}=\int_{B} \alpha \nabla u_{0} \cdot \nabla u_{1}
$$

By the characterization of the spectrum of an elliptic self-adjoint operator using the Rayleigh's quotient, we know that for all $v$ in $\mathrm{H}_{0}^{1}(\Omega)$ orthogonal to the first eigenfunction $u_{0}$, it holds that

$$
\lambda^{1} \int_{\Omega} v^{2} \leq \alpha \int_{\Omega}|\nabla v|^{2}
$$

where $\lambda^{1}>\lambda_{0}$ is the second eigenvalue of $-\alpha \Delta$ in $\mathrm{H}_{0}^{1}(\Omega)$. We have used the superscript here to distinguish the second eigenvalue $\lambda^{1}$ from $\lambda_{1}$ which appears in the second term of the expansion (2.1). Since $u_{1}$ is orthogonal to $u_{0}$, it follows using (2.16) that

$$
\alpha\left(1-\frac{\lambda_{0}}{\lambda^{1}}\right) \int_{\Omega}\left|\nabla u_{1}\right|^{2} \leq \int_{\Omega} \alpha\left|\nabla u_{1}\right|^{2}-\lambda_{0} \int_{\Omega} u_{1}^{2} \leq \alpha\left\|u_{0}\right\|_{\mathrm{H}_{0}^{1}(\Omega)}\left\|u_{1}\right\|_{\mathrm{H}_{0}^{1}(\Omega)}
$$

where at the end we have used (2.13) and followed it by a simple estimation. We have obtained the upper bound for $u_{1}$. Finally, using the variational characterization of the first eigenvalue for elliptic self-adjoint operators, we obtain

$$
\begin{aligned}
\lambda_{0}=\int_{\Omega} \alpha\left|\nabla u_{0}\right|^{2} \leq \int_{\Omega} \alpha\left|\nabla u_{\varepsilon}\right|^{2} & \leq \int_{\Omega} \alpha\left(1+\chi_{B} \varepsilon\right)\left|\nabla u_{\varepsilon}\right|^{2}=\lambda_{\varepsilon} \\
& \leq \int_{\Omega} \alpha\left(1+\chi_{B} \varepsilon\right)\left|\nabla u_{0}\right|^{2} \leq(1+\varepsilon) \int_{\Omega} \alpha\left|\nabla u_{0}\right|^{2}=(1+\varepsilon) \lambda_{0}
\end{aligned}
$$

which allows us to conclude that that $\left|\lambda_{\varepsilon}-\lambda_{0}\right| \leq C \varepsilon$. 
To use the quasimode strategy, we compute:

$$
\begin{aligned}
-\operatorname{div}( & \left.\alpha\left(1+\chi_{B} \varepsilon\right) \nabla\left(u_{0}+\varepsilon u_{1}\right)\right)-\left(\lambda_{0}+\varepsilon \lambda_{1}\right)\left(u_{0}+\varepsilon u_{1}\right) \\
= & -\alpha \Delta u_{0}-\lambda_{0} u_{0}+\varepsilon\left(-\alpha \Delta u_{1}-\lambda_{0} u_{1}-\lambda_{1} u_{0}-\operatorname{div}\left(\alpha \chi_{B} \nabla u_{0}\right)\right) \\
& \quad+\varepsilon^{2}\left(-\lambda_{1} u_{1}-\operatorname{div}\left(\alpha \chi_{B} \nabla u_{1}\right)\right) \\
= & \varepsilon^{2}\left(-\lambda_{1} u_{1}-\operatorname{div}\left(\alpha \chi_{B} \nabla u_{1}\right)\right)
\end{aligned}
$$

where we have used (2.4) and, (2.6) with $i=1$.

Proof of Proposition 2.1: We need a uniform bound on the normalized right-hand side: $\lambda_{1} u_{1}-$ $\operatorname{div}\left(\chi_{B} \nabla u_{1}\right)$. Obviously, this term is only defined in $\mathrm{H}^{-1}(\Omega)$ hence we have to make the estimation in the $\mathrm{H}^{-1}(\Omega)$ norm. To that end, we use a test function $\varphi \in \mathrm{H}_{0}^{1}(\Omega)$ and compute the duality product:

$$
\begin{aligned}
\left\langle-\operatorname{div}\left(\alpha \chi_{B} \nabla u_{1}\right), \varphi\right\rangle_{\mathrm{H}^{-1}(\Omega) \times \mathrm{H}_{0}^{1}(\Omega)} & =\int_{\Omega} \alpha \chi_{B} \nabla u_{1} \cdot \nabla \varphi=\int_{B} \alpha \nabla u_{1} \cdot \nabla \varphi \\
& \leq \alpha\left\|u_{1}\right\|_{\mathrm{H}_{0}^{1}(\Omega)}\|\varphi\|_{\mathrm{H}_{0}^{1}(\Omega)} .
\end{aligned}
$$

This proves that

$$
\left\|-\operatorname{div} \alpha\left(\chi_{B} \nabla u_{1}\right)\right\|_{\mathrm{H}^{-1}(\Omega)} \leq \alpha\left\|u_{1}\right\|_{\mathrm{H}_{0}^{1}(\Omega)} .
$$

And

$$
\begin{aligned}
\left\langle\lambda_{1} u_{1}, \varphi\right\rangle_{\mathrm{H}^{-1}(\Omega) \times \mathrm{H}_{0}^{1}(\Omega)} & =\int_{\Omega} \lambda_{1} u_{1} \varphi \leq \lambda_{1}\left\|u_{1}\right\|_{\mathrm{L}^{2}(\Omega)}\|\varphi\|_{\mathrm{L}^{2}(\Omega)} \\
& \leq \lambda_{1}\left\|u_{1}\right\|_{\mathrm{H}_{0}^{1}(\Omega)}\|\varphi\|_{\mathrm{H}_{0}^{1}(\Omega)} \leq C\|\varphi\|_{\mathrm{H}_{0}^{1}(\Omega)}
\end{aligned}
$$

using the estimation $\left(2.12\right.$ and the fact that $u_{1}$ is bounded independently of $B$ proved in Lemma 2.4 . This gives

$$
\left\|\lambda_{1} u_{1}\right\|_{\mathrm{H}^{-1}(\Omega)} \leq C .
$$

Hence, we obtain from (2.18), using (2.19) and (2.20) that there exists a constant $C$ independent of $B$ such that

$$
\left\|-\operatorname{div}\left(\alpha\left(1+\chi_{B} \varepsilon\right) \nabla\left(u_{0}+\varepsilon u_{1}\right)\right)-\left(\lambda_{0}+\varepsilon \lambda_{1}\right)\left(u_{0}+\varepsilon u_{1}\right)\right\|_{H^{-1}(\Omega)} \leq C \varepsilon^{2}
$$

Moreover, using $u_{0} \in \mathrm{H}_{0}^{1}$ as test function in the definition of the $\mathrm{H}^{-1}$-norm of $u_{0}+\varepsilon u_{1}$, we obtain

$$
\begin{aligned}
\left\|u_{0}+\varepsilon u_{1}\right\|_{\mathrm{H}^{-1}(\Omega)} & =\sup _{\varphi \in \mathrm{H}_{0}^{1}(\Omega)} \frac{\left\langle u_{0}+\varepsilon u_{1}, \varphi\right\rangle_{\mathrm{H}^{-1}, \mathrm{H}_{0}^{1}}}{\|\varphi\|_{\mathrm{H}_{0}^{1}(\Omega)}}=\sup _{\varphi \in \mathrm{H}_{0}^{1}(\Omega)} \frac{\int_{\Omega}\left(u_{0}+\varepsilon u_{1}\right) \varphi}{\|\varphi\|_{\mathrm{H}_{0}^{1}(\Omega)}} \\
& \geq \frac{\int_{\Omega}\left(u_{0}+\varepsilon u_{1}\right) u_{0}}{\left\|u_{0}\right\|_{\mathrm{H}_{0}^{1}(\Omega)}}=\frac{\int_{\Omega} u_{0}^{2}}{\left(\int_{\Omega}\left|\nabla u_{0}\right|^{2}\right)^{\frac{1}{2}}}=\sqrt{\frac{\alpha}{\lambda_{0}}}
\end{aligned}
$$

Hence, by 2.21 and 2.22, we obtain

$$
\left\|-\operatorname{div}\left(\alpha\left(1+\chi_{B} \varepsilon\right) \nabla\left(u_{0}+\varepsilon u_{1}\right)\right)-\left(\lambda_{0}+\varepsilon \lambda_{1}\right)\left(u_{0}+\varepsilon u_{1}\right)\right\|_{\mathrm{H}^{-1}(\Omega)} \leq \sqrt{\frac{\lambda_{0}}{\alpha}} C \varepsilon^{2}\left\|u_{0}+\varepsilon u_{1}\right\|_{\mathrm{H}^{-1}(\Omega)}
$$


As a consequence of the theory of quasi mode, there is an element of the spectrum of the self-adjoint operator $-\operatorname{div}\left(\alpha\left(1+\chi_{B} \varepsilon\right) \nabla \cdot\right)$ in $\mathrm{H}^{-1}(\Omega)$ at distance at most $\sqrt{\frac{\lambda_{0}}{\alpha}} C \varepsilon^{2}$ from $\lambda_{0}+\varepsilon \lambda_{1}$. To finish, we need to argue that this element of the spectrum is $\lambda_{\varepsilon}$, the first eigenvalue of $-\operatorname{div}\left(\alpha\left(1+\chi_{B} \varepsilon\right) \nabla \cdot\right)$. If these were higher eigenvalues, then as $\varepsilon \rightarrow 0$, they would tend to a higher eigenvalue of the operator $-\alpha \Delta$. But this would lead to a contradiction, since this sequence is within a distance $O\left(\varepsilon^{2}\right)$ from the sequence $\lambda_{0}+\varepsilon \lambda_{1}$ which tends to $\lambda_{0}$, the first eigenvalue of $-\alpha \Delta$ which is simple.

\section{Remainder of order two}

We first prove an uniform upper bound for $\lambda_{2}$ and $u_{2}$.

Lemma 2.5 There exists $C$, which is independent of $B$, such that:

$$
\left\|u_{2}\right\|_{\mathrm{H}_{0}^{1}(\Omega)} \leq C \text { and } \lambda_{2} \leq C .
$$

Proof of Lemma 2.5: First, notice that by 2.7 applied with $i=2$, we get

$$
\lambda_{2}=\int_{B} \alpha \nabla u_{0} \cdot \nabla u_{1} \leq \alpha\left\|u_{0}\right\|_{\mathrm{H}_{0}^{1}(\Omega)}\left\|u_{1}\right\|_{\mathrm{H}_{0}^{1}(\Omega)} \leq C
$$

where $C$ is independent of $B$ by the estimate (2.11). In a second step, we search a uniform estimate for $u_{2}$. To that end, we follow the strategy already used to estimate $u_{1}$. The main change is that $u_{2}$ is not orthogonal to $u_{0}$ so the adaptation is not straightforward. To overcome the difficulty we introduce the combination $u_{2}+a u_{0}$ where

$$
a=-\int_{\Omega} u_{2} u_{0}
$$

is chosen such that $u_{2}+a u_{0}$ is $\mathrm{L}^{2}(\Omega)$-orthogonal to $u_{0}$.

By (2.8) for $i=2$ we have

$$
\int_{\Omega} u_{2} u_{0}=-\frac{1}{2} \int_{\Omega} u_{1}^{2}
$$

which gives

$$
a=\frac{1}{2} \int_{\Omega} u_{1}^{2} \leq \frac{1}{2}\left\|u_{1}\right\|_{\mathrm{H}^{1}(\Omega)}^{2} \leq C
$$

with $C$ independent of $B$ (by (2.11)). We now estimate $u_{2}+a u_{0}$. For this, we multiply equation (2.4) by $a$ and add it to equation 2.6 to obtain:

$$
\begin{aligned}
-\alpha \Delta\left(u_{2}+a u_{0}\right)-\lambda_{0}\left(u_{2}+a u_{0}\right) & =\operatorname{div}\left(\alpha \chi_{B} \nabla u_{1}\right)+\lambda_{1} u_{1}+\lambda_{2} u_{0}, \quad \text { in } \Omega \\
u_{2}+a u_{0} & =0 \text { on } \partial \Omega
\end{aligned}
$$

Using $u_{2}+a u_{0}$ as test function, it follows that

$$
\begin{aligned}
\alpha \int_{\Omega} \mid & \left.\nabla\left(u_{2}+a u_{0}\right)\right|^{2}-\lambda_{0} \int_{\Omega}\left(u_{2}+a u_{0}\right)^{2} \\
& =\int_{B} \alpha \nabla u_{1} \cdot \nabla\left(u_{2}+a u_{0}\right)+\int_{\Omega} \lambda_{1} u_{1}\left(u_{2}+a u_{0}\right)+\int_{\Omega} \lambda_{2} u_{0}\left(u_{2}+a u_{0}\right) \\
& \leq\left(\alpha\left\|u_{1}\right\|_{\mathrm{H}_{0}^{1}(\Omega)}+\lambda_{1}\left\|u_{1}\right\|_{\mathrm{H}_{0}^{1}(\Omega)}+\left|\lambda_{2}\right|\left\|u_{0}\right\|_{\mathrm{H}_{0}^{1}(\Omega)}\right)\left\|u_{2}+a u_{0}\right\|_{\mathrm{H}_{0}^{1}(\Omega)} \\
& \leq C\left\|u_{2}+a u_{0}\right\|_{\mathrm{H}_{0}^{1}(\Omega)}
\end{aligned}
$$


where $C$ is independent of $B$, by estimates (2.12), (2.11) and (2.24). Since $u_{2}+a u_{0}$ is orthogonal to $u_{0}$, similarly as in the estimation (2.17), we conclude that $u_{2}+a u_{0}$ is bounded in $\mathrm{H}_{0}^{1}(\Omega)$ uniformly in $B$. Therefore,

$$
\left\|u_{2}\right\|_{\mathrm{H}_{0}^{1}(\Omega)} \leq C+a\left\|u_{0}\right\|_{\mathrm{H}_{0}^{1}(\Omega)} \leq C^{\prime}
$$

with $C^{\prime}$ independent of $B$ by estimate 2.26 .

Proof of Proposition 2.2; $\quad$ We compute

$$
\begin{aligned}
- & \operatorname{div}\left(\alpha\left(1+\chi_{B} \varepsilon\right) \nabla\left(u_{0}+\varepsilon u_{1}+\varepsilon^{2} u_{2}\right)\right)-\left(\lambda_{0}+\varepsilon \lambda_{1}+\varepsilon^{2} \lambda_{2}\right)\left(u_{0}+\varepsilon u_{1}+\varepsilon^{2} u_{2}\right) \\
= & -\alpha \Delta u_{0}-\lambda_{0} u_{0}+\varepsilon\left(-\alpha \Delta u_{1}-\lambda_{0} u_{1}-\lambda_{1} u_{0}-\operatorname{div}\left(\alpha \chi_{B} \nabla u_{0}\right)\right) \\
& +\varepsilon^{2}\left(-\alpha \Delta u_{2}-\lambda_{0} u_{2}-\lambda_{1} u_{1}-\lambda_{2} u_{0}-\operatorname{div}\left(\alpha \chi_{B} \nabla u_{1}\right)\right) \\
& +\varepsilon^{3}\left(-\lambda_{1} u_{2}-\lambda_{2} u_{1}-\operatorname{div}\left(\alpha \chi_{B} \nabla u_{2}\right)\right)+\varepsilon^{4}\left(\lambda_{2} u_{2}\right) \\
= & \varepsilon^{3}\left(-\lambda_{1} u_{2}-\lambda_{2} u_{1}-\operatorname{div}\left(\alpha \chi_{B} \nabla u_{2}\right)\right)+\varepsilon^{4}\left(\lambda_{2} u_{2}\right)
\end{aligned}
$$

using equations (2.4), and 2.6 for $i=1,2$. Then, since

$$
\left\|-\operatorname{div}\left(\alpha \chi_{B} \nabla u_{2}\right)\right\|_{\mathrm{H}^{-1}(\Omega)} \leq \alpha\left\|u_{2}\right\|_{\mathrm{H}_{0}^{1}(\Omega)},
$$

it follows from equation (2.28) and estimates (2.12), (2.11), and (2.23), that for $\varepsilon \ll 1$,

$$
\begin{aligned}
& \left\|-\operatorname{div}\left(\alpha\left(1+\chi_{B} \varepsilon\right) \nabla\left(u_{0}+\varepsilon u_{1}+\varepsilon^{2} u_{2}\right)\right)-\left(\lambda_{0}+\varepsilon \lambda_{1}+\varepsilon^{2} \lambda_{2}\right)\left(u_{0}+\varepsilon u_{1}+\varepsilon^{2} u_{2}\right)\right\|_{\mathrm{H}^{-1}(\Omega)} \\
& \quad \leq\left(\left(\alpha+\lambda_{1}\right)\left\|u_{2}\right\|_{\mathrm{H}_{0}^{1}(\Omega)}+\left|\lambda_{2}\right|\left\|u_{1}\right\|_{\mathrm{H}_{0}^{1}(\Omega)}\right) \varepsilon^{3}+\left(\left|\lambda_{2}\right|\left\|u_{2}\right\|_{\mathrm{H}_{0}^{1}(\Omega)}\right) \varepsilon^{4} \\
& \quad \leq C_{1} \varepsilon^{3}+C_{2} \varepsilon^{4} \leq C \varepsilon^{3}
\end{aligned}
$$

Moreover, one has

$$
\begin{aligned}
\left\|u_{0}+\varepsilon u_{1}+\varepsilon^{2} u_{2}\right\|_{\mathrm{H}^{-1}(\Omega)} & =\sup _{\varphi \in \mathrm{H}_{0}^{1}(\Omega)} \frac{\int_{\Omega}\left(u_{0}+\varepsilon u_{1}+\varepsilon^{2} u_{2}\right) \varphi}{\|\varphi\|_{\mathrm{H}_{0}^{1}(\Omega)}} \geq \frac{\int_{\Omega}\left(u_{0}+\varepsilon u_{1}+\varepsilon^{2} u_{2}\right) u_{0}}{\left\|u_{0}\right\|_{\mathrm{H}_{0}^{1}(\Omega)}} \\
& =\frac{\int_{\Omega} u_{0}^{2}+\varepsilon^{2} \int_{\Omega} u_{0} u_{2}}{\left\|u_{0}\right\|_{\mathrm{H}_{0}^{1}(\Omega)}} .
\end{aligned}
$$

Then, using relation 2.25, we obtain

$$
\left\|u_{0}+\varepsilon u_{1}+\varepsilon^{2} u_{2}\right\|_{\mathrm{H}^{-1}(\Omega)} \geq \frac{1-\frac{\varepsilon^{2}}{2} \int_{\Omega} u_{1}^{2}}{\left\|u_{0}\right\|_{\mathrm{H}_{0}^{1}(\Omega)}} \geq \frac{1-\frac{\varepsilon^{2}}{2} C^{2}}{\left\|u_{0}\right\|_{\mathrm{H}_{0}^{1}(\Omega)}},
$$

since $u_{1}$ is bounded in $H_{0}^{1}(\Omega)$ and consequently, in $L^{2}(\Omega)$ as shown in 2.11. For $\varepsilon<\frac{1}{C}$, we get

$$
\left\|u_{0}+\varepsilon u_{1}+\varepsilon^{2} u_{2}\right\|_{\mathrm{H}^{-1}(\Omega)} \geq \frac{1}{2\left\|u_{0}\right\|_{\mathrm{H}_{0}^{1}(\Omega)}}=\frac{1}{2} \sqrt{\frac{\alpha}{\lambda_{0}}}
$$


By (2.29) and (2.30), we then have for $\varepsilon<1 / C$ small enough

$$
\begin{aligned}
& \left\|-\operatorname{div}\left(\alpha\left(1+\chi_{B} \varepsilon\right) \nabla\left(u_{0}+\varepsilon u_{1}+\varepsilon^{2} u_{2}\right)\right)-\left(\lambda_{0}+\varepsilon \lambda_{1}+\varepsilon^{2} \lambda_{2}\right)\left(u_{0}+\varepsilon u_{1}+\varepsilon^{2} u_{2}\right)\right\|_{\mathrm{H}^{-1}(\Omega)} \\
& \quad \leq 2 C \varepsilon^{3} \sqrt{\frac{\lambda_{0}}{\alpha}}\left\|u_{0}+\varepsilon u_{1}+\varepsilon^{2} u_{2}\right\|_{\mathrm{H}^{-1}(\Omega)} .
\end{aligned}
$$

By the quasimode argument, there is an element of the spectrum of $-\operatorname{div}\left(\alpha\left(1+\chi_{B} \varepsilon\right) \nabla \cdot\right)$ in $H^{-1}(\Omega)$ whose distance from $\lambda_{0}+\varepsilon \lambda_{1}+\varepsilon^{2} \lambda_{2}$ is atmost $2 C \varepsilon^{3} \sqrt{\frac{\lambda_{0}}{\alpha}}$. By similar arguments as those at the end of Proposition 2.1. one concludes that such an element is precisely $\lambda_{\varepsilon}$, the first eigenvalue of $-\operatorname{div}\left(\alpha\left(1+\chi_{B} \varepsilon\right) \nabla \cdot\right)$.

\section{Minimization of the second order approximation of $\lambda(B)$}

Although our main interest is to minimize the ground state $\lambda_{\varepsilon}$ with respect to the set $B$, given $\varepsilon>0$, the general feeling is that the optimization problem is not well posed. A relaxed problem which is not so simple to describe was obtained in Cox and Lipton [6]. In order to understand the nature of the problem for small contrasts Conca et. al. used a first order approximation [3. Indeed, after proving a slightly weaker estimate as compared to Proposition 2.1 using a more ad hoc method of estimation, they conclude that

$$
\left|\inf _{B} \lambda_{\varepsilon}(B)-\lambda_{0}-\varepsilon \inf _{B} \lambda_{1}(B)\right| \leq C \varepsilon^{\frac{3}{2}}
$$

This permits to obtain approximate minimizers for the eigenvalue functional $\lambda_{\varepsilon}$ by minimizing, instead, the functional $\lambda_{0}+\varepsilon \lambda_{1}$. This is a well posed problem and since the original problem may not be well posed it may fail to capture some of the features of the original minimization problem. With this motivation, we go further and do a second order approximation. Indeed, Proposition 2.2 allows us to conclude that

$$
\left|\inf _{B} \lambda_{\varepsilon}(B)-\inf _{B}\left(\lambda_{0}+\varepsilon \lambda_{1}(B)+\varepsilon^{2} \lambda_{2}(B)\right)\right| \leq C \varepsilon^{3} .
$$

Thus, we can obtain approximate minimizers for the functional $\lambda_{\varepsilon}$, for given $\varepsilon>0$ small enough, by minimizing the functional $\lambda_{0}+\varepsilon \lambda_{1}+\varepsilon^{2} \lambda_{2}$ which is a second order approximation of $\lambda_{\varepsilon}$. We then study the problem:

$$
\operatorname{minimize}\left\{\lambda_{0}+\varepsilon \lambda_{1}(B)+\varepsilon^{2} \lambda_{2}(B) ; B \subseteq \Omega,|B|=m\right\}, \quad 0<m<|\Omega|, m \text { fixed }
$$

or equivalently

$$
\text { minimize }\left\{\lambda_{1}(B)+\varepsilon \lambda_{2}(B) ; B \subseteq \Omega,|B|=m\right\},
$$

since $\lambda_{0}$ is independent of $B$ and $\varepsilon>0$ is fixed. From the expressions for $\lambda_{1}(B), \lambda_{2}(B)$ computed in the previous section, we finally consider the problem

$$
\operatorname{minimize} F(\chi):=\alpha \int_{\Omega} \chi\left(\nabla u_{0}+\varepsilon \nabla v(\chi)\right) \cdot \nabla u_{0}
$$

over the class of admissible domains represented by their characteristic functions

$$
\mathcal{U}_{a d}:=\left\{\chi ; \chi=\chi_{B}, B \subseteq \Omega,|B|=m\right\} \subseteq \mathrm{L}^{\infty}(\Omega),
$$

and $v=v(\chi) \in \mathrm{H}_{0}^{1}(\Omega)$ satisfisfies

$$
\begin{aligned}
-\alpha \Delta v-\lambda_{0} v=\lambda_{1}(\chi) u_{0} & +\operatorname{div}\left(\alpha \chi \nabla u_{0}\right), \\
\lambda_{1}(\chi) & :=\int_{\Omega} \alpha \chi\left|\nabla u_{0}\right|^{2}, \\
v & \perp u_{0} \text { in } L^{2}(\Omega) .
\end{aligned}
$$




\subsection{Relaxation of the minimization problem}

The functional $F$ is lower-semicontinuous for the weak-* topology on $L^{\infty}(\Omega)$, being quadratic with respect to $\chi$, but the admissible set $\mathcal{U}_{a d}$ is not closed for this topology. In order to have a well-posed minimization problem we need to work on the closure $\overline{\mathcal{U}_{a d}}$ and calculate the lower semicontinuous envelope of $F$ with respect to the weak-* topology on $L^{\infty}(\Omega)$.

$$
\bar{F}(\theta):=\inf \left\{\liminf F\left(\chi_{n}\right): \chi_{n} \rightarrow \theta \operatorname{in} \mathrm{L}^{\infty}(\Omega)^{*}\right\}, \theta \in \overline{\mathcal{U}_{a d}},
$$

where

$$
\overline{\mathcal{U}_{a d}}=\overline{\mathcal{U}}_{a d}{ }^{\infty}(\Omega)^{*}=\left\{\theta \in L^{\infty}(\Omega) ; 0 \leq \theta \leq 1, \int_{\Omega} \theta=m\right\} .
$$

We shall follow the general procedure to compute $\bar{F}$ and obtain the following theorem.

Theorem 3.1 For any $\theta \in \overline{\mathcal{U}_{a d}}$, we have

$$
\bar{F}(\theta)=\alpha \int_{\Omega} \theta\left[\nabla u_{0}+\varepsilon \nabla v_{\infty}(\theta)\right] . \nabla u_{0}-\varepsilon \theta(1-\theta)\left|\nabla u_{0}\right|^{2},
$$

where $v_{\infty}(\theta) \in \mathrm{H}_{0}^{1}(\Omega)$ is solution of

$$
\begin{aligned}
-\alpha \Delta v-\lambda_{0} v=\lambda_{1}(\theta) u_{0} & +\operatorname{div}\left(\alpha \theta \nabla u_{0}\right), \\
\lambda_{1}(\theta) & :=\int_{\Omega} \alpha \theta\left|\nabla u_{0}\right|^{2}, \\
v & \perp u_{0} \text { in } \mathrm{L}^{2}(\Omega) .
\end{aligned}
$$

The proof of the Theorem 3.1 will use some results on $H$-measures. This tool was introduced by P. Gérard in [11] and L. Tartar in [16] to understand the obstruction to compactness via a matrix of complex-valued Radon measures $\left(\mu_{i j}(x, \xi)\right)_{1 \leq i, j \leq p}$ on $\mathbb{R}^{N} \times \mathbb{S}^{N-1}$ on the space-frequency domain associated to weakly convergent sequences. We refer to the two previous references for a complete presentation of $H$-measures and to [1] for their applications in small contrast homogenization. We will need the two following results (Theorem 2-2 and Lemma 2-3 in [1]).

Theorem 3.2 [1] Let $u_{\varepsilon}$ be a sequence which weakly converges to 0 in $\mathrm{L}^{2}\left(\mathbb{R}^{N}\right)^{p}$. There exists a subsequence and a $H$-measure $\mu$ such that

$$
\lim _{\varepsilon \rightarrow 0} \int_{\mathbb{R}^{N}} q\left(u_{\varepsilon}\right) \cdot \bar{u}_{\varepsilon}=\int_{\mathbb{R}^{N}} \int_{\mathbb{S}^{N-1}} \sum_{i, j=1}^{p} q_{i j}(x, \xi) \mu_{i j}(d x, d \xi)
$$

for any polyhomogeneous pseudo-differential operator $q$ of degree 0 with symbol $\left(q_{i j}(x, \xi)\right)$.

We shall also use the following lemma due to Kohn and Tartar that deals with the special case of sequences of characteristic functions.

Lemma 3.3 [1] Let $\chi_{\varepsilon}$ be a sequence of characteristic functions that weakly-* converges to some $\theta$ in $\mathrm{L}^{\infty}(\Omega,[0,1])$. Then the corresponding $H$-measure $\mu$ for the sequence $\left(\chi_{\varepsilon}-\theta\right)$ is necessarily of the type

$$
\mu(d x, d \xi)=\theta(x)(1-\theta(x)) \nu(d x, d \xi)
$$

where, for a given $x$, the measure $\nu(d x, d \xi)$ is a probability measure with respect to $\xi$.

Conversely, for any such probability measure $\nu \in \mathcal{P}\left(\Omega, \mathbb{S}^{N-1}\right)$, there exists a sequence $\chi_{\varepsilon}$ of characteristic functions which weakly-* converges to $\theta \in \mathrm{L}^{\infty}(\Omega,[0,1])$ such that $\theta(1-\theta) \nu$ is the $H$-measure of $\left(\chi_{\varepsilon}-\theta\right)$. 
Proof of Proposition 3.1: Let $\theta \in \overline{\mathcal{U}_{a d}}$. Let $\left\{\chi_{n}\right\}$ be a sequence in $\mathcal{U}_{a d}$ such that

$$
\chi_{n} \stackrel{\star}{\rightarrow} \theta \in \overline{\mathcal{U}_{a d}} .
$$

We then analyze the limit of

$$
F\left(\chi_{n}\right)=\alpha \underbrace{\int_{\Omega} \chi_{n}\left|\nabla u_{0}\right|^{2}}_{A_{n}}+\alpha \varepsilon \underbrace{\int_{\Omega} \chi_{n} \nabla v_{n} \cdot \nabla u_{0}}_{B_{n}},
$$

with $v_{n}:=v\left(\chi_{n}\right) \in \mathrm{H}_{0}^{1}(\Omega)$ such that

$$
\begin{array}{r}
-\alpha \Delta v_{n}-\lambda_{0} v_{n}=\lambda_{1}\left(\chi_{n}\right) u_{0}+\operatorname{div}\left(\alpha \chi_{n} \nabla u_{0}\right), \\
\lambda_{1}\left(\chi_{n}\right)=\int_{\Omega} \alpha \chi_{n}\left|\nabla u_{0}\right|^{2}, \\
v_{n} \perp u_{0} \text { in } L^{2}(\Omega) .
\end{array}
$$

SteP 1: Passing to the limit in $A_{n}$ is easy. By the convergence (3.7), we have

$$
A_{n}=\lambda_{1}\left(\chi_{n}\right) \longrightarrow \alpha \int_{\Omega} \theta\left|\nabla u_{0}\right|^{2}=\lambda_{1}(\theta)
$$

Step 2: Now we study the limit of the sequence $v_{n}$. By $(3.9)$, we know that

$$
\left(1-\frac{\lambda_{0}}{\lambda^{1}}\right) \int_{\Omega}\left|\nabla v_{n}\right|^{2} \leq C
$$

using a similar estimation as 2.17 . Then $\left\|v_{n}\right\|_{\mathrm{H}_{0}^{1}}^{2} \leq C$ and hence,

$$
v_{n} \rightarrow v_{\infty}=v_{\infty}(\theta) \quad \text { weak- } \mathrm{H}_{0}^{1}(\Omega)
$$

up to a subsequence. Since $\mathrm{H}_{0}^{1}(\Omega)$ is compactly embedded in $\mathrm{L}^{2}(\Omega)$,

$$
v_{n} \longrightarrow v_{\infty} \quad \text { in } \mathrm{L}^{2}(\Omega)
$$

up to a subsequence. Therefore, we can pass to variational limit from 3.8 to obtain,

$$
-\alpha \Delta v_{\infty}-\lambda_{0} v_{\infty}=\lambda_{1}(\theta) u_{0}+\operatorname{div}\left(\alpha \theta \nabla u_{0}\right)
$$

Moreover, passing to the limit from (3.9), we have

$$
v_{\infty} \perp u_{0} \text { in } \mathrm{L}^{2}(\Omega)
$$

accordingly, since $\left\|u_{0}\right\|_{\mathrm{L}^{2}}=1, v_{\infty}=v_{\infty}(\theta)$ is uniquely defined in (3.11) and $v_{\infty}$ depends (linearly) only on $\theta$ and not on the convergent subsequence of $\left\{v_{n}\right\}$.

Step 3: The main difficulty is to pass to the limit in $B_{n}$ which is quadratic with respect to $\chi_{n}$. First, we can rewrite $B_{n}$ as

$$
B_{n}=\int_{\Omega} \chi_{n} \nabla w_{n} \cdot \nabla u_{0}+\int_{\Omega} \chi_{n} \nabla z_{n} \cdot \nabla u_{0},
$$

$w_{n}, z_{n} \in \mathrm{H}_{0}^{1}(\Omega)$ such that

$$
-\alpha \Delta w_{n}=\lambda_{0} v_{n}+\lambda_{1}\left(\chi_{n}\right) u_{0}
$$




$$
-\Delta z_{n}=\operatorname{div}\left(\chi_{n} \nabla u_{0}\right)
$$

On the one hand, since

$$
\lambda_{0} v_{n}+\lambda_{1}\left(\chi_{n}\right) u_{0} \longrightarrow \lambda_{0} v_{\infty}+\lambda_{1}(\theta) u_{0} \quad \text { in } L^{2}(\Omega),
$$

(3.13) implies

$$
w_{n} \longrightarrow w \quad \text { in } \mathrm{H}_{0}^{1}(\Omega)
$$

where $w \in \mathrm{H}_{0}^{1}(\Omega)$ satisfies the equation

$$
-\alpha \Delta w=\lambda_{0} v_{\infty}+\lambda_{1}(\theta) u_{0}
$$

and, in consequence,

$$
\int_{\Omega} \chi_{n} \nabla w_{n} \cdot \nabla u_{0} \longrightarrow \int_{\Omega} \theta \nabla w \cdot \nabla u_{0} .
$$

The difficulty is now to calculate the limit in the second term of $B_{n}$ in $(3.12)$. We observe that div $\chi_{n} \nabla u_{0} \rightarrow$ $\operatorname{div} \theta \nabla u_{0}$ weakly in $\mathrm{H}^{-1}(\Omega)$ and since $(-\Delta)^{-1}$ is a isomorphism from $\mathrm{H}^{-1}(\Omega)$ into $\mathrm{H}_{0}^{1}(\Omega)$, we get $\mathrm{L}^{2}$-weak convergence of $\nabla z_{n}$. However, this is not enough for passing to the limit in the second term of $B_{n}$ because, in the product $\chi_{n} \nabla z_{n}$, both sequences $\chi_{n}$ and $\nabla z_{n}$ only converge weakly. For handling this convergence problem we use the results on $H$-convergence stated before.

STEP 4: For simplicity if $\Omega$ is $\mathbb{R}^{n}$, in view of Theorem 3.2 and Lemma 3.3 , the limit of the second term in $(3.12)$ becomes

$$
\lim _{n \rightarrow \infty} \int_{\mathbb{R}^{N}} \chi_{n} \nabla z_{n} \cdot \nabla u_{0}=\int_{\mathbb{R}^{N}} \theta Q(\theta) \cdot \nabla u_{0}-\int_{\mathbb{R}^{N}} \theta(1-\theta) M \nabla u_{0} \cdot \nabla u_{0},
$$

where the pseudo-differential operator $Q$ is defined in Lemma 5.1 (in the appendix) and it's symbol has been calculated therein and,

$$
M=\int_{\mathbb{S}^{N-1}} \xi \otimes \xi \nu(x, \mathrm{~d} \xi),
$$

$\nu=\nu(x, \xi)$ is a probability measure with respect to $\xi$ that depends on the sequence $\left\{\chi_{n}\right\}$ and $Q(\theta)=\nabla z$ with $z \in \mathrm{H}_{0}^{1}(\Omega)$ verifies the equation

$$
-\Delta z=\operatorname{div}\left(\theta \nabla u_{0}\right) \text {. }
$$

SteP 5: But we need to work on $\Omega$ bounded. To that end, we use a localization procedure. This argument proceeds as follows. Let $\left(\zeta_{k}\right)$ be a sequence of smooth compactly supported functions in $C_{0}^{\infty}\left(\mathbb{R}^{N}\right)$ such that $\operatorname{supp} \zeta_{k} \subset \Omega$ for all $k$ and $\zeta_{k}$ converges to 1 strongly in $\mathrm{L}^{2}(\Omega)$. Then the second term on the right hand side of $(3.12)$ can be written as

$$
\int_{\Omega} \chi_{n} \nabla z_{n} \cdot \nabla u_{0}=\int_{\mathbb{R}^{N}} \zeta_{k} \chi_{n} \nabla z_{n} \cdot \nabla u_{0}+\int_{\mathbb{R}^{N}}\left(1-\zeta_{k}\right) \chi_{n} \nabla z_{n} \cdot \nabla u_{0}
$$

Note that the last term in (3.16) converges to 0 uniformly with respect to $n$ when $k$ tends to infinity because $z_{n}$ is bounded in $\mathrm{H}^{1}(\Omega)$. We now fix $k$ and consider another smooth compactly supported function $\psi_{k} \in C_{0}^{\infty}$ such that $\psi_{k} \equiv 1$ inside the support of $\zeta_{k}$. The first term on the right hand side of $(3.16)$ is thus equal to

$$
\int_{\Omega} \zeta_{k}\left(\psi_{k} \chi_{n}\right) \nabla\left(\psi_{k} z_{n}\right) \cdot \nabla u_{0}
$$

Rewriting the equation 3.14 in $\mathbb{R}^{N}$ as

$$
-\Delta\left(\psi_{k} z_{n}\right)-\Delta\left(\left(1-\psi_{k}\right) z_{n}\right)=\operatorname{div}\left(\psi_{k} \chi_{n} \nabla u_{0}\right)+\operatorname{div}\left(\left(1-\psi_{k}\right) \chi_{n} \nabla u_{0}\right),
$$


we can show that the function $\psi_{k} z_{n}$ is the sum of $\tilde{z}_{n}, \check{z}_{n}$ on the support of $\zeta_{k}$ being $\tilde{z}_{n}, \check{z}_{n}$ solutions of the following equations in the whole space $\mathbb{R}^{N}$

$$
\begin{aligned}
-\Delta \tilde{z}_{n} & =\operatorname{div} \psi_{k} \chi_{n} \nabla u_{0} \quad \text { in } \mathbb{R}^{N}, \\
\Delta \check{z}_{n} & =\operatorname{div} z_{n} \nabla \psi_{k}+\nabla \psi_{k} \cdot\left(\chi_{n} \nabla u_{0}+\nabla z_{n}\right) \quad \text { in } \mathbb{R}^{N} .
\end{aligned}
$$

We then notice that

$\operatorname{div} \psi_{k} \chi_{n} \nabla u_{0} \rightarrow \operatorname{div} \psi_{k} \theta \nabla u_{0} \quad$ weakly in $\mathrm{H}^{-1}\left(\mathbb{R}^{N}\right)$ and

$$
\operatorname{div} z_{n} \nabla \psi_{k}+\nabla \psi_{k} \cdot\left(\chi_{n} \nabla u_{0}+\nabla z_{n}\right) \rightarrow \operatorname{div} z \nabla \psi_{k}+\nabla \psi_{k} \cdot\left(\theta \nabla u_{0}+\nabla z\right) \quad \text { strongly in } \mathrm{H}^{-1}\left(\mathbb{R}^{N}\right)
$$

since this last term clearly converges weak- $\mathrm{L}^{2}(\Omega)$. Using the fact that $(-\Delta)^{-1}$ is an isomorphism from $\mathrm{H}^{-1}\left(\mathbb{R}^{N}\right)$ into $H^{1}\left(\mathbb{R}^{N}\right)$, we thus have

$$
\tilde{z}_{n} \rightarrow \tilde{z} \quad \text { weakly in } H^{1}\left(\mathbb{R}^{N}\right)
$$

and

$$
\check{z}_{n} \rightarrow \check{z} \quad \text { strongly in } H^{1}\left(\mathbb{R}^{N}\right)
$$

where $\tilde{z}, \check{z}$ verify

$$
\begin{aligned}
-\Delta \tilde{z} & =\operatorname{div} \psi_{k} \theta \nabla u_{0} \quad \text { in } \mathbb{R}^{N}, \\
\Delta \check{z} & =\operatorname{div} z \nabla \psi_{k}+\nabla \psi_{k} \cdot\left(\theta \nabla u_{0}+\nabla z\right) \quad \text { in } \mathbb{R}^{N} .
\end{aligned}
$$

Obviously $z=\tilde{z}+\check{z}$ on the support of $\zeta_{k}$.

Now noting that the integral (3.17) has close relationship with the formulation of the $H$-measures, we see that, as in the whole space case, $\nabla \tilde{z}_{n}$ depends linearly on $\left(\psi_{k} \chi_{n}\right)$ through the pseudo-differential operator $Q$ of symbol (5.2). Therefore applying Theorem 2 of [11], we conclude that the limit of the first term on the right hand side of $(3.16)$ is equal to

$$
\begin{aligned}
\lim _{n \rightarrow \infty} \int_{\mathbb{R}^{N}} \zeta_{k}\left(\psi_{k} \chi_{n}\right) \nabla\left(\check{z}_{n}+\tilde{z}_{n}\right) \cdot \nabla u_{0} & =\int_{\mathbb{R}^{N}} \zeta_{k}\left(\psi_{k} \theta\right) \nabla \check{z} \cdot \nabla u_{0} \\
& +\lim _{n \rightarrow \infty} \int_{\mathbb{R}^{N}} \zeta_{k}\left(\psi_{k} \chi_{n}\right) \nabla \tilde{z}_{n} \cdot \nabla u_{0} \\
=\int_{\mathbb{R}^{N}} \zeta_{k}\left(\psi_{k} \theta\right) \nabla \check{z} \cdot \nabla u_{0} & +\int_{\mathbb{R}^{N}} \zeta_{k}\left(\psi_{k} \theta\right) \nabla \tilde{z} \cdot \nabla u_{0}-\int_{\mathbb{R}^{N}} \zeta_{k} \psi_{k} \theta(1-\theta) M \nabla u_{0} \cdot \nabla u_{0} \\
& =\int_{\Omega} \zeta_{k} \theta \nabla z \cdot \nabla u_{0}-\int_{\Omega} \zeta_{k} \theta(1-\theta) M \nabla u_{0} \cdot \nabla u_{0} .
\end{aligned}
$$

Finally making $k$ tends to $\infty$, we obtain the desired bounded domain case.

We go back to the calculation of the limit in (3.12). Indeed, gathering the limit (3.15) and limit calculated above, it follows that

$$
\lim _{n \rightarrow \infty} B_{n}=\int_{\Omega} \theta \nabla v_{\infty}(\theta) \cdot \nabla u_{0}-\int_{\Omega} \theta(1-\theta) \int_{\mathbb{S}^{N-1}}\left(\xi \cdot \nabla u_{0}\right)^{2} \nu(\mathrm{d} x, \mathrm{~d} \xi) .
$$

From (3.10) and (3.18), finally one has

$$
\begin{aligned}
\lim _{n \rightarrow \infty} F\left(\chi_{n}\right) & =\lim _{n \rightarrow \infty} A_{n}+\varepsilon \lim _{n \rightarrow \infty} B_{n} \\
& =\frac{1}{\alpha} \lambda_{1}(\theta)+\varepsilon \int_{\Omega} \theta \nabla v_{\infty}(\theta) \cdot \nabla u_{0}-\varepsilon \int_{\Omega} \theta(1-\theta) \int_{\mathbb{S}^{N-1}}\left(\xi \cdot \nabla u_{0}\right)^{2} \nu(\mathrm{d} x, \mathrm{~d} \xi) .
\end{aligned}
$$


STEP 6: Now we calculate

$$
\bar{F}(\theta)=\inf _{\nu} \lim F\left(\chi_{n}\right)
$$

To that end, we notice that

$$
\int_{\mathbb{S}^{N-1}}\left(\xi \cdot \nabla u_{0}\right)^{2} \nu(\mathrm{d} x, \mathrm{~d} \xi) \leq\left|\nabla u_{0}\right|^{2}(x) \quad \text { a.e. } x \in \Omega
$$

since $\nu$ is a probability measure with respect to $\xi$ a.e. $x \in \Omega$. Moreover, this value is reached when we take the Dirac measure $\delta_{\nabla u_{0}(x)}$, i.e., when

$$
\nu(x, \xi)=\delta_{\xi_{x}} \mathrm{~d} x, \quad \xi_{x}=\nabla u_{0}(x) .
$$

From the converse part of Lemma 2.3 in [1] the minimum for

$$
\inf _{\nu} \lim F\left(\chi_{n}\right)
$$

is also achieved. So, finally we can conclude

$$
\bar{F}(\theta)=\int_{\Omega} \theta\left(\nabla u_{0}+\varepsilon \nabla v_{\infty}(\theta)\right) \cdot \nabla u_{0}-\varepsilon \int_{\Omega} \theta(1-\theta)\left|\nabla u_{0}\right|^{2} .
$$

Recall that $v_{\infty}=v_{\infty}(\theta)$ depends linearly on $\theta$.

\subsection{Optimality conditions for the relaxed problem.}

The relaxed functional $\bar{F}$ achieves it's minimum of $\overline{\mathcal{U}_{a d}}$ since it is lower-semicontinuous and the constraint set is compact for the weak-* topology. We first investigate the differentiability properties of $\bar{F}$ in order to obtain optimality conditions for a minimizer of $\bar{F}$ on the compact convex set $\overline{\mathcal{U}_{a d}}$.

Proposition 3.4 The functional $\bar{F}$ is Fréchet differentiable of every order and we have the following expressions for the Gateaux derivatives of first and second order

$$
\bar{F}^{\prime}(\theta) \varphi=\int_{\Omega}\left[2 \varepsilon\left(\nabla v_{\infty}(\theta)+\theta \nabla u_{0}\right)+(1-\varepsilon) \nabla u_{0}\right] \cdot \nabla u_{0} \varphi
$$

and

$$
\bar{F}^{\prime \prime}(\theta)(\varphi, \varphi)=2 \varepsilon \int_{\Omega}\left(\nabla v_{\infty}(\varphi)+\varphi \nabla u_{0}\right) \cdot \nabla u_{0} \varphi
$$

Proof: The linearity of the application $\theta \mapsto v_{\infty}(\theta)$ and the expression for $\bar{F}$ show clearly that it is quadratic with respect to $\theta$. So, the Fréchet derivatives exist. In order to calculate the first order derivative, we rewrite 3.19 as

$$
\bar{F}(\theta)=\varepsilon \int_{\Omega} \theta \nabla v_{\infty}(\theta) \cdot \nabla u_{0}+\varepsilon \int_{\Omega} \theta^{2}\left|\nabla u_{0}\right|^{2}+(1-\varepsilon) \int_{\Omega} \theta\left|\nabla u_{0}\right|^{2} .
$$

But, using $v_{\infty}(\theta)$ as test function in (3.11), we get

$$
\bar{F}(\theta)=-\varepsilon \int_{\Omega}\left|\nabla v_{\infty}(\theta)\right|^{2}-\frac{\lambda_{0}}{\alpha} v_{\infty}^{2}(\theta)+\varepsilon \int_{\Omega} \theta^{2}\left|\nabla u_{0}\right|^{2}+(1-\varepsilon) \int_{\Omega} \theta\left|\nabla u_{0}\right|^{2} .
$$

A simple calculation gives us

$$
\bar{F}^{\prime}(\theta) \varphi=-2 \varepsilon \int_{\Omega} \nabla v_{\infty}(\theta) \cdot \nabla v_{\infty}(\varphi)-\frac{\lambda_{0}}{\alpha} v_{\infty}(\theta) v_{\infty}(\varphi)+2 \varepsilon \int_{\Omega} \theta\left|\nabla u_{0}\right|^{2} \varphi+(1-\varepsilon) \int_{\Omega}\left|\nabla u_{0}\right|^{2} \varphi
$$

We now notice that $v_{\infty}(\varphi)$ satisfies (3.11). Then, again taking $v_{\infty}(\theta)$ as test function, we can explicitly write the above expression in terms of $\varphi$ to obtain (3.20) then (3.21). 
We wish to investigate the critical points for the constrained minimization problem of minimizing $\bar{F}$ over $\overline{\mathcal{U}_{a d}}$. To that end, we use the Lagrange's multipliers method with the constraint

$$
C(\theta):=\int_{\Omega} \theta=m, \quad \theta \in \overline{\mathcal{U}_{a d}} \text { hence } C^{\prime}(\theta) \varphi=\int_{\Omega} \varphi .
$$

Therefore, the critical points satisfy the Euler-Lagrange equation: for all admissible $\varphi$

$$
\left[\bar{F}^{\prime}(\theta)+\Lambda C^{\prime}(\theta)\right] \varphi=0
$$

for some $\Lambda \in \mathbb{R}$; i.e.

$$
\int_{\Omega}\left[2 \varepsilon\left(\nabla v_{\infty}(\theta)+\theta \nabla u_{0}\right)+(1-\varepsilon) \nabla u_{0}\right] \cdot \nabla u_{0} \varphi+\Lambda \int_{\Omega} \varphi=0 \quad \forall \varphi .
$$

Consequently the density of $\overline{\mathcal{U}_{a d}}$ in $\mathrm{L}^{2}(\Omega)$ implies

$$
2 \varepsilon \nabla v_{\infty}(\theta) \cdot \nabla u_{0}+(2 \varepsilon \theta+1-\varepsilon)\left|\nabla u_{0}\right|^{2}=\Lambda \quad \text { on } \Omega .
$$

Proposition 3.5 If $\theta^{*}$ is optimal in the relaxed formulation, then there is real $\Lambda$ such that:

$$
2 \varepsilon \nabla v_{\infty}(\theta) \cdot \nabla u_{0}+(2 \varepsilon \theta+1-\varepsilon)\left|\nabla u_{0}\right|^{2}=\Lambda \quad \text { in } \Omega .
$$

Integrating over $\Omega$ and considering $u_{0}$ as test function in (3.11), we get the following consequence

$$
\int_{\Omega} \nabla v_{\infty}(\theta) \cdot \nabla u_{0}=0 \text { and } 2 \varepsilon \int_{\Omega} \theta\left|\nabla u_{0}\right|^{2}+\frac{1-\varepsilon}{\alpha} \lambda_{0}=\Lambda|\Omega| .
$$

\section{Numerical illustrations}

In this section we shall illustrate the behavior of the solution of the appoximated problem through numerical simulations. To that end, we place ourselves under assumption of low contrast regime, i.e. $\beta=\alpha(1+\varepsilon)$ for small $\varepsilon$. In the following examples, we will consider $\varepsilon=0.1$ and $\varepsilon=10^{-6}$.

We use an optimization algorithm to minimize $\bar{F}$ : we have implemented a gradient-based steepest descend numerical algorithm for the local proportion $\theta$. At each step of the optimization algorithm, we update the local proportion with a step $\rho_{i}>0$ by

$$
\theta_{i}=\min \left(1, \max \left(0, \tilde{\theta}_{i}\right)\right) \text { with } \tilde{\theta}_{i}=\theta_{i-1}-\rho_{i} \bar{F}^{\prime}\left(\theta_{i-1}\right)+\Lambda_{i}
$$

where $\Lambda_{i}$ is the Lagrange multiplier for the volume constraint. The Lagrange multipliers $\Lambda_{i}$ are approximated at each iteration by simple dichotomy in order to get the constraint $\int_{\Omega} \theta_{i}=m$ corresponding to a fixed proportion.

The optimization procedure is coupled with finite elements approximations of the boundary values problems needed to compute both $\bar{F}$ and its derivative $\bar{F}^{\prime}$. To calculate the eigen-pair $\left(\lambda_{0}, u_{0}\right)$ and all the states $v_{i, \infty}$, we use $\mathbb{P}_{2}$ finite elements while the local proportions $\theta_{i}$ have been discretized with $\mathbb{P}_{1}$.

We will present examples in dimension two and three. The computations have been made with the FEM library FreeFem++ [12]. The subsequent figures show the local proportion of the material with higher conductivity. We do a comparative analysis in dimension two and three for square and cube cases respectively confirming the mentioned properties in [3] with respect to the distribution of the material with higher conductivity that depends on the shape of the domain $\Omega$. The volume always refers to the percentage of volume occupied by the higher conductivity material. 


\subsection{The square and the cube.}

The computations are made on the unit square $[0,1]^{2}$ with a regular mesh of 80000 triangles. For a very small value of $\varepsilon$ here $10^{-6}$, we have obtained the optimal designs displayed into Figure 4.1 for different volume proportions. The dark red region corresponds to $B$ and material $\beta$, the local proportion is then 1 . The blue region correspond to material $\alpha$, the local proportion is then 0 .

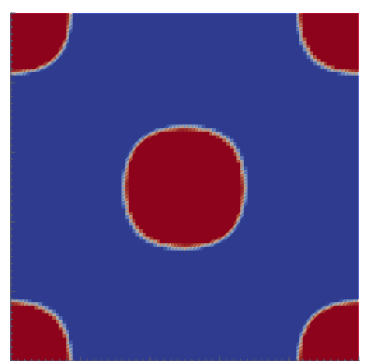

(a) $m /|\Omega|=0.2$

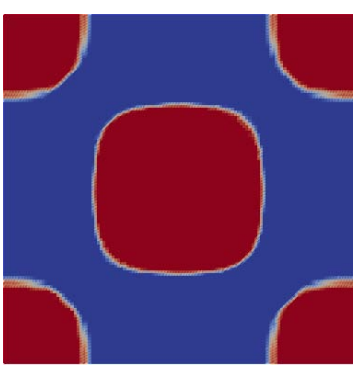

(b) $m /|\Omega|=0.4$

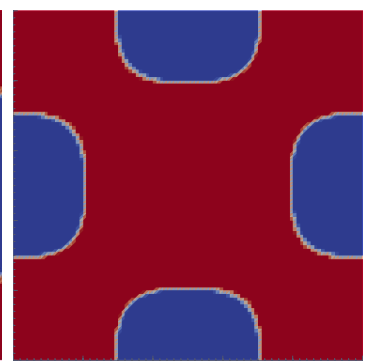

(c) $m /|\Omega|=0.7$

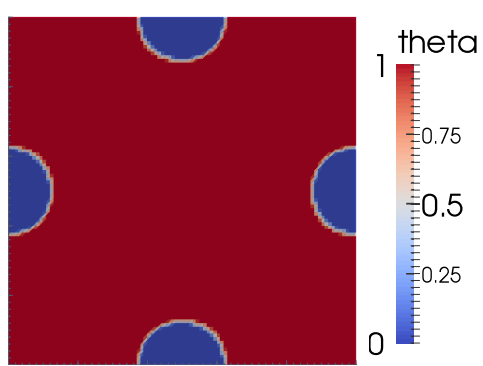

(d) $m /|\Omega|=0.9$

Figure 4.1: Nearly optimal distribution $B$ in the square case for $\varepsilon=10^{-6}$.

The numerically computed optimal region $B$ contains neighborhoods around corners and the center always is also included. Similar results were obtained by Conca, Laurain and Mahadevan in [3] with a first order approximation only. Nevertheless, the local proportion is very often either 0 either 1 . Let us now consider the same cases with a much larger parameter $\varepsilon$. In Figure 4.2, we present the results obtained with $\varepsilon=0.1$. We observe that the mixture is much more important: there seems to be a pure material nowhere. We believe that the asymptotic is not reached for such a large value of $\varepsilon$.

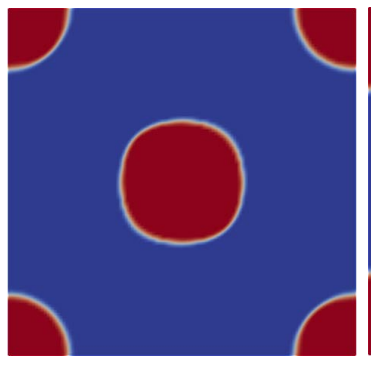

(a) $m /|\Omega|=0.2$

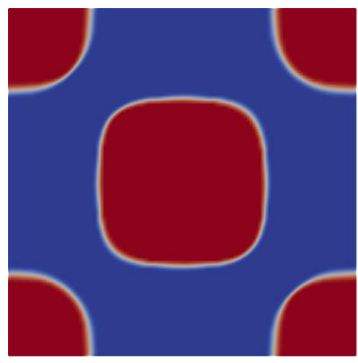

(b) $m /|\Omega|=0.4$

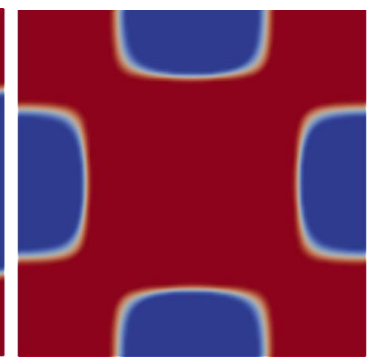

(c) $m /|\Omega|=0.7$

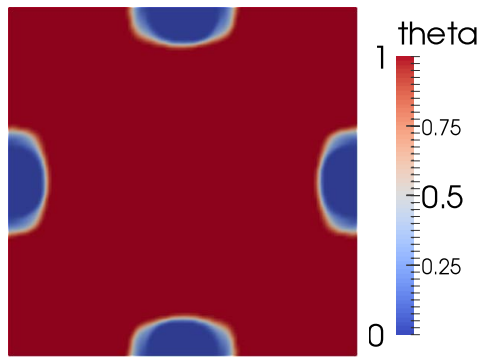

(d) $m /|\Omega|=0.9$

Figure 4.2: Nearly optimal distribution $B$ in the square case for $\varepsilon=0.1$.

Let us now present simulations on the unit cube $[0,1]^{3}$. For the visualisation, we have remove the phase where $\theta=0$. Since the computation have been made on a Laptop, the resolution is coarser in these simulations in dimension three, we kept the same numbers of degree of freedom.

\subsection{Others domains}

For the sake of completeness, we present computations in other plane domains for the comparison with [3]: a crescent in Figure 4.4 and a perforated ellipse in Figure 4.5 .

Let us emphasize that in the last case, even for $\varepsilon=10^{-6}$, we observe clearly in Figure 4.5(c) a small area where $\theta$ takes values strictly between 0 and 1 where we see the effect of the modelling with a second order approximation. 


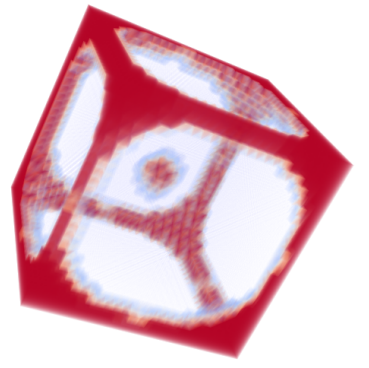

(a) $m /|\Omega|=0.125$

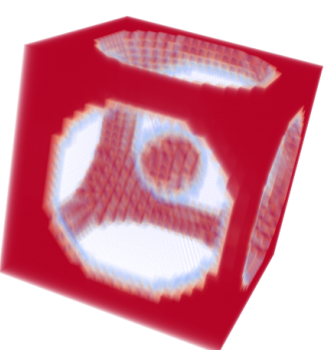

(b) $m /|\Omega|=0.25$

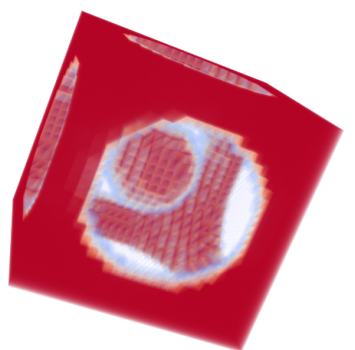

(c) $m /|\Omega|=0.375$

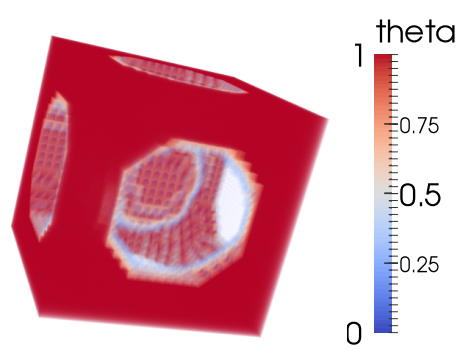

(d) $m /|\Omega|=0.5$

Figure 4.3: Nearly optimal distribution $B$ in the cube case for $\varepsilon=10^{-6}$.

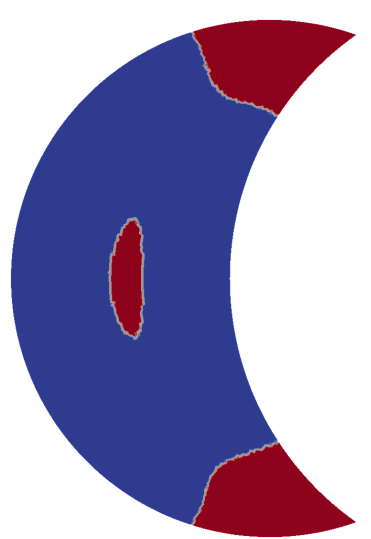

(a) $m /|\Omega|=0.2$

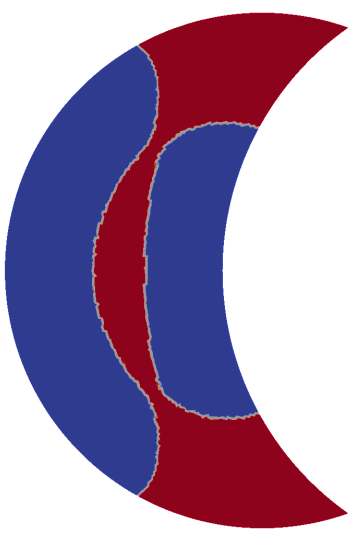

(b) $m /|\Omega|=0.4$

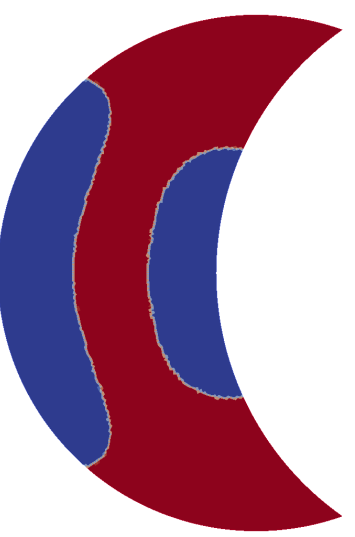

(c) $m /|\Omega|=0.6$

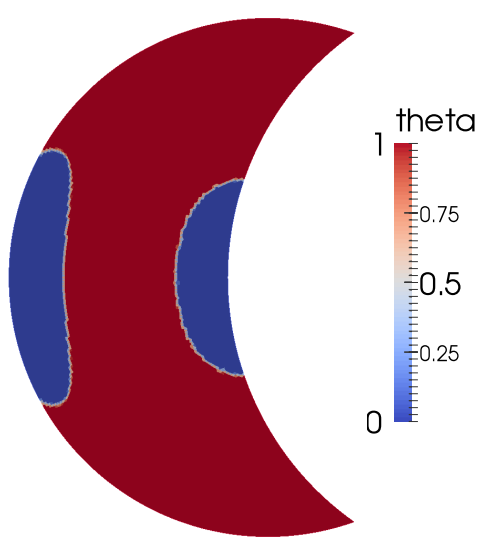

(d) $m /|\Omega|=0.8$

FiguRE 4.4: Nearly optimal distribution $B$ in a crescent for $\varepsilon=10^{-6}$.

\section{Appendix}

Lemma 5.1 For $\theta \in L^{\infty}(\Omega)$, if $z \in H_{0}^{1}(\Omega)$ solves

$$
-\Delta z=\operatorname{div}\left(\theta \nabla u_{0}\right) \quad \text { in } \mathbb{R}^{N},
$$

then $\theta \mapsto Q(\theta):=\nabla z$ defines a pseudo-differential operator with symbol

$$
q(x, \xi)=-\frac{\xi \cdot \nabla u_{0}(x)}{|\xi|^{2}} \xi
$$

Note that $q$ is homogenous of degree 0 in $\xi$.

Proof of Lemma 5.1: We first consider the whole space case in order to use Fourier calculus. Indeed, denoting by ${ }^{\wedge}$ the Fourier transform and starting from Equation (3.14), formally we can calculate as follows

$$
\begin{aligned}
(-\Delta z)^{\Upsilon}(\xi) & =\left(\operatorname{div}\left(\theta \nabla u_{0}\right)\right)^{\uparrow}(\xi) \\
-\left(-|\xi|^{2} \widehat{z}\right) & =-i \xi \cdot \nabla u_{0}(x) \widehat{\theta} \\
\widehat{z} & =-\frac{i \xi \cdot \nabla u_{0}(x)}{|\xi|^{2}} \widehat{\theta},
\end{aligned}
$$




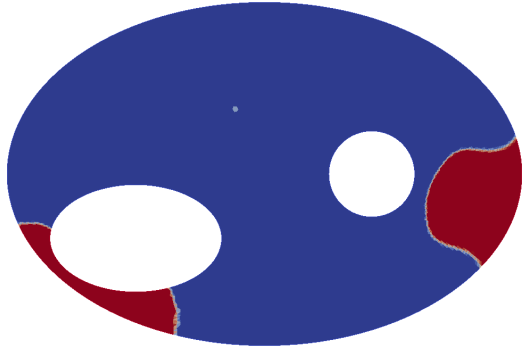

(a) $m /|\Omega|=0.1$

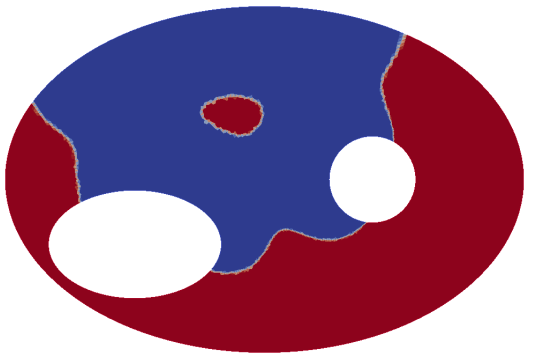

(d) $m /|\Omega|=0.5$

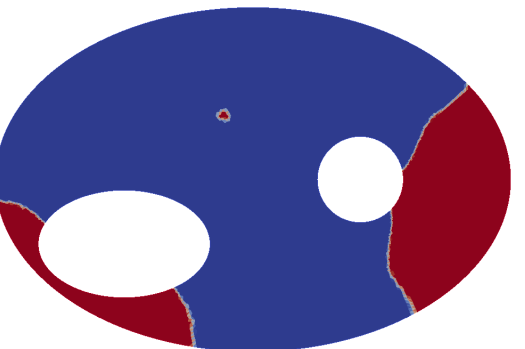

(b) $m /|\Omega|=0.2$

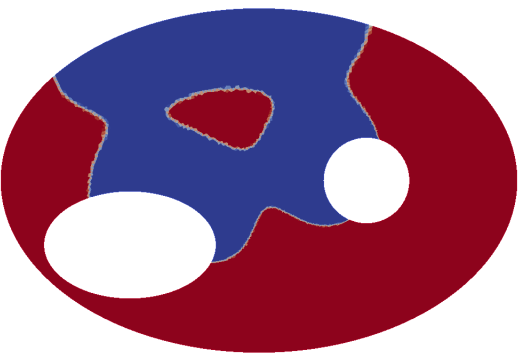

(e) $m /|\Omega|=0.6$

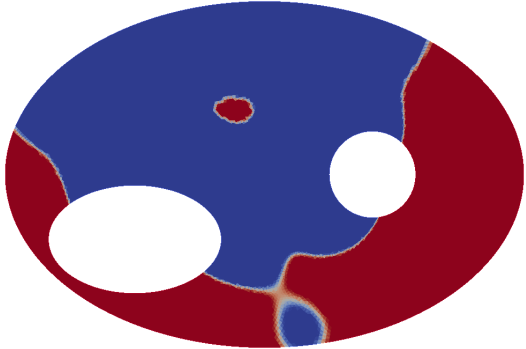

(c) $m /|\Omega|=0.4$

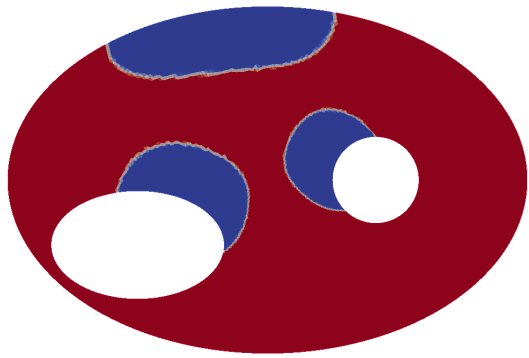

(f) $m /|\Omega|=0.8$

FiguRE 4.5: Nearly optimal distribution $B$ in a perforated ellipse for $\varepsilon=10^{-6}$.

which gives

$$
\widehat{\nabla z}(\xi)=-i \xi \widehat{z}(\xi)=-i \xi\left(-\frac{i \xi \cdot \nabla u_{0}(x)}{|\xi|^{2}} \widehat{\theta}\right)=\underbrace{\left(-\frac{\xi \cdot \nabla u_{0}(x)}{|\xi|^{2}} \xi\right)}_{q(x, \xi)} \widehat{\theta} .
$$

Acknowledgements: The authors thank support from ECOS-CONICYT Grant C13 05. The first author is also partially supported by Fondecyt Grant $\mathrm{N}^{\circ} 1140773$. The second author is also partially supported by the ANR Grant ARAMIS and OPTIFORM. The last author acknowledges the support of Fondecyt Grant No 1130595.

\section{References}

[1] Grégoire Allaire and Sergio Gutiérrez. Optimal design in small amplitude homogenization. M2AN Math. Model. Numer. Anal., 41(3):543-574, 2007.

[2] A. Alvino, G. Trombetti, and P.-L. Lions. On optimization problems with prescribed rearrangements. Nonlinear Anal., 13(2):185-220, 1989.

[3] Carlos Conca, Antoine Laurain, and Rajesh Mahadevan. Minimization of the ground state for two phase conductors in low contrast regime. SIAM J. Appl. Math., 72(4):1238-1259, 2012.

[4] Carlos Conca, Rajesh Mahadevan, and León Sanz. An extremal eigenvalue problem for a two-phase conductor in a ball. Appl. Math. Optim., 60(2):173-184, 2009. 
[5] Carlos Conca, Rajesh Mahadevan, and Leon Sanz. Shape derivative for a two-phase eigenvalue problem and optimal configurations in a ball. In CANUM 2008, volume 27 of ESAIM Proc., pages 311-321. EDP Sci., Les Ulis, 2009.

[6] Steven Cox and Robert Lipton. Extremal eigenvalue problems for two-phase conductors. Arch. Rational Mech. Anal., 136(2):101-117, 1996.

[7] Steven Cox and JR McLaughlin. Extremal eigenvalue problems for composite membranes i. Appl. Math. Optim., 22(2):153-167, 1990.

[8] Steven Cox and JR McLaughlin. Extremal eigenvalue problems for composite membranes ii. Appl. Math. Optim., 22(2):169-187, 1990.

[9] M. Dambrine and D. Kateb. On the shape sensitivity of the first Dirichlet eigenvalue for two-phase problems. Appl. Math. Optim., 63(1):45-74, 2011.

[10] M. Dauge, I. Djurdjevic, E. Faou, and A. Rössle. Eigenmode asymptotics in thin elastic plates. J. Math. Pures Appl. (9), 78(9):925-964, 1999.

[11] Patrick Gérard. Microlocal defect measures. Comm. Partial Differential Equations, 16(11):1761-1794, 1991.

[12] F. Hecht. New development in freefem++. J. Numer. Math., 20(3-4):251-265, 2012.

[13] Antoine Henrot. Extremum problems for eigenvalues of elliptic operators. Frontiers in Mathematics. Birkhäuser Verlag, Basel, 2006.

[14] Antoine Laurain. Global minimizer of the ground state for two phase conductors in low contrast regime. ESAIM: Control, Optimisation and Calculus of Variations, 20:362-388, 42014.

[15] Franz Rellich. Perturbation theory of eigenvalue problems. Assisted by J. Berkowitz. With a preface by Jacob T. Schwartz. Gordon and Breach Science Publishers, New York, 1969.

[16] Luc Tartar. $H$-measures, a new approach for studying homogenisation, oscillations and concentration effects in partial differential equations. Proc. Roy. Soc. Edinburgh Sect. A, 115(3-4):193-230, 1990.

\section{Carlos Conca}

Universidad de Chile

Department of Engineering Mathematics, Center for Mathematical Modelling (CMM), UMI 2807 CNRSChile \& Center for Biotechnology and Bioengineering (CeBiB), Universidad de Chile, Santiago, Chile.

E-mail: cconca@dim.uchile.cl

Marc Dambrine

Département de Mathématiques, Université de Pau et des Pays de l'Adour

E-mail: marc.dambrine@univ-pau.fr

Rajesh Mahadevan 
Depto. de Matemática, Fac. Cs. Fís. y Matemáticas, Universidad de Concepción

E-mail: rmahadevan@udec.cl

\section{Duver Quintero}

Université de Pau et des Pays de l'Adour \& Universidad de Chile.

E-mail: duver@dim.uchile.cl 